\title{
The collateral benefits of having chiropractic available in a public central hospital
}

\author{
J an Roar Orlin ${ }^{1}$, Andrè Didriksen², Helge Hagen ${ }^{3}$, Anders Sørfonden ${ }^{4}$ \\ 1. Dept. of Orthopedics, Central Hospital (FSS), Førde, Norway. 2. Private clinic, Førde, Norway. 3. Dept. of Ear- \\ Nose-Throat, Central Hospital (FSS), Førde, Norway. 4. Lærdal Hospital, Norway.
}

Correspondence: Jan Roar Orlin. Address: Tårnveien 26, 1430 Ås, Norway. Email: ja-or@online.no

Received: May 15, 2013

DOI : $10.5430 /$ jha.v2n4p138

Accepted: July 25, 2013

URL: http://dx.doi.org/10.5430/jha.v2n4p138

Online Published: August 8, 2013

\begin{abstract}
Following previous reports on the co-operation between a chiropractor and a central hospital, experiences from the past five years are presented. The objective of this paper is to show that improved management of muscular and skeletal problems within a hospital setting depends on the availability of chiropractic health care as a treatment option. The following pain groups were sampled: 1) sacro-lumbar dysfunction and sciatic leg symptoms, with or without joint dysfunction and sciatica; 2) myo-fascial referred pain syndromes, frequently caused by peripheral nerve entrapment; and 3) tinnitus, dizziness/vertigo, facial pain, ear plug and swallowing difficulties, frequently caused by biomechanical components. A majority of pain patients, after being subjected to traditional conservative treatment, usually over a period of several years, fail to return to work despite younger than average age. The only effective procedures seem to be those of chiropractors. In order to benefit from their particular knowledge, public hospitals need to open their doors to chiropractors. For that to happen, determined hospital administrators are needed.
\end{abstract}

\section{Key words}

Chiropractic, Leg pain, Functional based spinal lesions

\section{Introduction}

Patients with muscular and skeletal problems may become a burden to National Health budgets. In public hospitals, highly-specialized teams offer evidence-based medicine and modern diagnostic procedures and treatments to patients with widespread muscle pain. Many specialties are involved. Yet, the national costs of muscular and skeletal pain seem unaffected by these interventions, and are steadily increasing. Pain clinics, composed of multiple specialty groups, were instituted to offer solutions to patients incapacitated by all kinds of muscular and skeletal pain, but in practice they frequently do not meet expectations. In this setting, orthopedic surgeons have become extremely sub-specialized, often treating degenerative joint disease and fractures in only one part of the body, whereas chiropractors treat functional lesions in the entire body. Unfortunately, the interest of the medical profession in understanding chiropractic principles has not been high.

Traditionally, hospital medical staffs have considered the treatment offered by chiropractors to be of dubious value and not grounded on evidence-based research. However, chiropractic care now has a growing body of evidence-based research ${ }^{[1]}$. Leading chiropractic teaching institutions in the world have science-based educational programs comparable to those of 
medical teaching institutions. Research by Triano in 1992 led to definition of the basic concept of a functional spinal lesion ${ }^{[2]}$. When the interaction between the nervous system, facet joints with their movement receptors and the musculature is disturbed, a functional spinal lesion follows. A neuro-biomechanical approach to restore normal balance created by monosynaptic reflexes acting between joints and the deep spinal muscles is needed. With patients placed in a prone or supine position to relax them, soft tissue techniques, aimed at affecting muscle tone at the site of primary joint lesions and at secondary compensatory lesions, are used. These techniques include pressure on local muscular pain locations (= trigger points), often in combination with passive stretching and traction to achieve relaxation. The main treatment consists of joint adjustment techniques which is a fast passive stretching of deep structures around the joint, with the utmost importance placed on appropriate force, speed, amplitude and movement specificity.

In a few universities in some countries, medical and chiropractic students spend their first years under the same roof. The result has not only been mutual acknowledgement, but also a new and exciting combination of medical doctor and chiropractor. In recognition of chiropractors' ability to reduce muscular-skeletal pain and increase function, Norwegian chiropractors in 2006 were given the right to report patients sick and to refer them to specialists. Otherwise, the possible advantages of muscular and skeletal pain reduction and cost benefits from closer cooperation between chiropractors and hospital staffs have been given few incentives. In Norway, two areas may contribute to improvements: emerging plans to start educating chiropractors at one of the universities and large-scale research into diseases causing muscular and skeletal pain, with financing, initiated by the Norwegian Department of Health ${ }^{[3]}$.

Geographically-close relationships and joint cooperation between chiropractic and hospital medicine is the first condition for improving the situation. For patients and hospitals, all arguments seem to speak in favor of incorporating chiropractors into daily routines. In view of the myriad landscape of natural healers increasingly offering their services, such inclusion would appear as another important signal to the public that chiropractic is highly recommended by official health administrators.

\section{Orthopedic materials}

\subsection{Lumbo-sacral muscular fixation}

Patients experiencing great pain caused by acute functional lumbo-sacral fixation, resulting from combined lumbar flexion and rotation, usually arrive in orthopedic departments camouflaged as sciatica ${ }^{[4]}$. In the absence of neurological changes and space-occupying spinal lesions appearing on lumbar magnetic resonance imaging (MRI), orthopedic surgeons have nothing to offer but medication. This approach is mostly ineffective from a 2-week to 3-week perspective of slow mobilization led by physiotherapists. Instead, the treatment of choice should be chiropractic, most often leading to immediate mobilization ${ }^{[4]}$.

During the period from 2008 to 2011, 28 patients were treated within the Central Hospital of Sogn and Fjordane for functional lumbo-sacral fixation because they were unable to enter a taxi to visit a private chiropractic clinic. The average number of treatments given within the hospital was two, and four and a half were given until full restitution. Available information from the Norwegian National Health Service, department of statistics, states that without chiropractic treatment, patients belonging to this group are out of work for 72 days. Furthermore, $49 \%$ of patients who are out of work for more than 56 days (eight weeks) never return to their original jobs. A much larger number of hospitalized patients were discharged and then sent down town by taxi for ambulatory chiropractic treatment. They received an average of three treatments per week for two weeks. According to personal follow up on both patient groups, everyone returned to their job.

\subsubsection{First case report}

A 50 year old working family man, living a 15-minute drive from the hospital, experienced sudden lower lumbar pain while getting out of bed one morning. No one was allowed to touch him. A helicopter was needed for transport to the 
hospital, and general anesthesia was needed in order to move him into a hospital bed and to the orthopedic department. Clinical examination and lumbar MRI revealed no abnormalities other than a muscular lumbo-sacral trans-articular fixation. A chiropractor (coauthor A.D.) was summoned from his down town private practice. He provided standard treatment, after which the patient was able to immediately walk away. One follow-up treatment was performed.

\subsubsection{Second case report}

The most recent patient was a female medical doctor, aged 36 years, working in the hospital. While lifting and carrying an office chair, she experienced acute lumbo-sacral pain that was so intense that she was crawling. While trying to go upstairs, she got dizzy and syncopated for five seconds. Afterwards, she experienced involuntary bladder retention. Her pain was bilateral with total loss of weight-bearing capacity and movement, and she had to stay in bed. After confirming that her eight week pregnancy was unaffected, she was given $20 \mathrm{mg}$ of morphine twice that day. The next day, she regained full bladder control, but the pain was even worse than the day before. With all clinical tests negative, she was offered chiropractic treatment, which led to immediate ambulation. She had no need for sick leave. After three days of follow-up chiropractic treatment, her functioning was fully restored. Finally, she was referred to her local physiotherapist for further rehabilitation of her core musculature.

\subsection{Pain and referred pain caused by peripheral nerve entrapment}

Another group of patients often has widespread muscular pain in the arms and legs, and in the lumbar, thoracic, and cervical areas. Chiropractic often leads to temporary pain reduction only. These patients may return time and again with local pain as well as "referred muscular pain" with obviously tense and painful muscles, commonly caused by different peripheral nerve entrapments.

In the upper extremity, a frequent cause of peripheral nerve entrapment is the lacertus fibrosus, impinging upon the median nerve proximally in the lower arm. This phenomenon seems to occur frequently, but it is little known and rarely surgically treated, whereas the entrapment of the median nerve in the carpal tunnel is more familiar. Recently, a research project on this phenomenon was approved by the regional ethical committee ${ }^{[5]}$. Peripheral nerve entrapment in the arm is also known to cause "referred" pain in the shoulder ${ }^{[6,7]}$.

In the lower extremity, leg pain and cramps seem to accompany chronic compartment syndrome ${ }^{[8]}$, affecting nerves in the foot and leg compartments as well as in the tarsal tunnels. Due to the close working conditions in the region, both chiropractors and medical doctors are learning to recognize and refer these conditions to the orthopedic department, despite its apparent biomechanical presentation. Some chiropractic colleges have started to accept and teach these theories of pain and stiffness in the thoracic region ${ }^{[9]}$.

\section{Third case report}

Following recent heart surgery, a 53 year old woman approached the chiropractic clinic for treatment. Previously, she had experienced back ache and sciatica for 14 years. After heart surgery, she was instructed to walk for 1 hour- 2 hours daily as rehabilitation, but she was unable to walk 100 meters without getting bilateral leg pain and toe-cramps. The pain always dominated on her right side and the so called sciatica always became worse after walking, ending with excruciating leg and foot pain with cramps during the day as well as at night. The only relief could be achieved by walking slowly inside her apartment at night, leading to sleep loss, depression, and an additional psychiatric diagnosis. She had given up on these problems years ago because all treatment efforts had been ineffective.

The recognition of her signs and symptoms indicated chronic compartment syndrome of the legs and all tests were positive ${ }^{[8]}$. A recent MRI scan of her lumbar spinal canal revealed normal findings and she was referred to the orthopedic department for further examination and surgery ${ }^{[8]}$. Upon revisiting the chiropractic clinic three months later, her right leg had been operated on and she was waiting for the same procedure to be performed in the other leg. Being only half way through surgery, she described her result as "getting her life back" after 14 years of terrible quality of life. 


\subsection{Ear, nose and throat materials}

Following the positive cooperation experience between the orthopedic department and a chiropractor, another pre-study was performed with the ENT department of the same hospital ${ }^{[10]}$. Patients with tinnitus, facial pain, ear plug, swallowing difficulties, dizziness/vertigo, etc. were examined and treated by medical doctors only. After a full scale examination, many patients were frequently not given a specific diagnosis or acknowledged cause for treatment, but were prescribed medication. Cooperation between the ENT-department and a chiropractor showed that many patients' symptoms frequently had a biomechanical cause ${ }^{[10]}$. The ENT-specialists now check for biomechanical problems more often, but further research is needed, hopefully with funding from the hospital research department.

\section{Fourth case report}

A 54 year old woman had a history of left-sided facial pain, difficulty swallowing, and an ear plug, all on the same side. Most of these symptoms presented after an airplane flight 3 months previously. She had been sleeping, her head resting towards the window for about one hour. The next day she had full blown symptoms. She was not on sick-leave and could cope at work, but experienced increasing symptoms after office hours. Her general practitioner gave her pain killers, anti-inflammatories, and referred her to an ear, nose, and throat (ENT) specialist (co-author HH). A full scale examination showed no pathology. A slight torticollis and left-sided neck tightness was detected on palpation, and she was referred to the chiropractic clinic. The chiropractor found a left-sided combined upper cervical and jaw dysfunction, resulting in tension of her left mastication muscles. Her facial symptoms were reproduced by a hypertonic sterno-cleido-mastoideus muscle on the left side. Palpation of her left posterior digastric and stylo-hyoid muscles indicated a reason for her swallowing difficulties. These structures were functionally restored in the course of six treatments over two weeks until full restitution. It seems worth mentioning that ordinary cases of common cervical dysfunction imply 45 days of sick leave, according to the Norwegian National Health Service, department of statistics.

\section{Discussion}

The present study reports the benefits of close cooperation between a medical hospital staff and chiropractors. Major patient advantages have been achieved, particularly concerning cooperation with the orthopedic and ENT departments. Treatment of functional lumbo-sacral fixation with its accompanying sciatic-like pain, peripheral nerve entrapment with local and referred pain as well as tinnitus, facial pain, ear plug, swallowing difficulties and vertigo is illustrated by typical case reports.

\subsection{Educational benefits for hospitals}

In the absence of a close relationship between hospitals and chiropractors, medical students do not learn enough about the perspectives of chiropractic treatment to advise their patients later on. Twice a year, groups of 15 medical students from the University of Bergen, Norway, have spent two months of their clinical education at the Central Hospital of Sogn and Fjordane. Practically all students have attended a chiropractic treatment of a typical case belonging to the 28-patient group mentioned above, who were hospitalized due to acute lumbo-sacral fixation/dysfunction. Witnessing a bedridden patient achieving immediate mobilization illustrates the merits of chiropractic treatments better than words.

Another material of approximately 30 patients were remitted from one chiropractor (AD) to the Central Hospital in one year. Minor surgical treatment, like fasciotomy of all four leg compartments and decompression of peripheral nerves, has proven curative in these cases. Indications are that the presence of chronic compartment syndrome or tarsal tunnel syndrome predisposes to the development of sacro-iliac dysfunction. Surprisingly, both local and referred pain has disappeared in many patients treated for multi-entrapment. Even the tender points basic to a "diagnosis" of fibromyalgia disappeared along with the diagnosis. Ethically-approved research and separate reports on patient materials of these kinds are in production. 


\subsection{Benefits for chiropractors}

Besides the appreciation of being accepted and incorporated into a hospital's multi-educational environment, closer cooperation with hospital staff leads to better availability of services. In cases of sciatica, investigative imaging such as x-ray, computed tomography and MRI often represent the difference between satisfied and dissatisfied patients. A negative MRI supports chiropractic treatment for functional muscular pain whereas a positive MRI confirming a disc lesion prohibits chiropractic treatment for fear of causing an accidental increase in pain. Complaints from patients who have such experiences lead to poor publicity for chiropractors. Further support, such as laboratory tests, are also available as well as advice from neurologists, rheumatologists, colleagues in physical medicine, orthopedic surgeons and others.

Perhaps as a consequence of close relations between students of medicine and chiropractic at the South Danish University (SDU) in Odense, Denmark, by 2011 three Danes and two Norwegians graduated after studying chiropractic first and medicine second ${ }^{[11]}$. Currently, three additional Norwegian chiropractors are following the same path at the SDU, increasing the total number of combined chiropractor/medical doctors in Norway to five. This number might be expected to rise due to the attractive challenge of fulfilling two important roles within health care. Which experience could possibly be better fit for bridging the gap between chiropractic and medicine ${ }^{[12,13]}$ ?

\section{Conclusions}

The aim of the present case series is to illustrate the benefits of close cooperation between medical hospital staff and chiropractors, particularly the orthopedic and ENT departments. Patients' pain reduction as a result of seemingly untraditional diagnostic procedures and treatment of functional sacro-lumbar fixation, local and referred pain caused by multiple peripheral nerve entrapments, as well as patients experiencing tinnitus, facial pain, ear plug, swallowing difficulties, and dizziness/vertigo seems promising. In principle, doctors should handle structural lesions whilst chiropractors can solve functional based problems. The extent of potential savings in public budgets from large scale cooperation between modern chiropractic and medicine is yet to be determined. To succeed, it seems mandatory to incorporate chiropractors into hospitals and to welcome their views and abilities with an open mind and a positive attitude.

\section{Recommendation}

We recommend that the traditional multi-specialty pain clinic concept be extended to include chiropractors. For this to happen, energetic hospital administrators are needed.

\section{References}

[1] Bronfort G, Haas M, Evans R, Leininger B, Triano J. Effectiveness of manual therapies: the UK evidence report. Chiropractic and osteopathy. 2010; 18(3): 1-33.

[2] Triano JJ. Interaction of spinal biomechanics and physiology. In: Haldemann S, editor. Principles and practice of chiropractic. 2nd ed. Connecticut: Appleton \& Lange; 1992. pp. 225-257.

[3] National group for health research. Suggestions for national implementations on Muscle-and Skeletal Pain, Damages and Diseases (MSPSS). [Internet] October 6th 2011, pp. 1-21. Available from: www.Helseforsk.no

[4] Orlin JR, Didriksen A. Results of chiropractic treatment of lumbopelvic fixation in 44 patients admitted to an orthopaedic department. J Manipulative Physiol Ther. 2007; 30(2): 135-139. PMid: 17320735. http://dx.doi.org/10.1016/j.jmpt.2006.12.011

[5] Aasved H, Hjelmeland K, Orlin JR, Andersen JR, Mader K, Francziszty Z, et al. Entrapment of the median nerve by lacertus fibrosus proximally in the lower arm. A prospective analysis of pain and quality of life before and after operation. (Approved by the Regional Ethics'Committee June 2012).

[6] Cherington M. Proximal pain in carpal tunnel syndrome. Arch Surg. 1974; 108(1): 69. PMid: 4808578. http://dx.doi.org/10.1001/archsurg.1974.01350250059016

[7] Kummel BM, Zazanis GA. Shoulder pain as the presenting complaint in carpal tunnel syndrome. Clin Orthop. 1973; 92(2): 227-230. PMid: 4710836. http://dx.doi.org/10.1097/00003086-197305000-00019 
[8] Orlin JR, Øen J, Andersen JR. Changes in leg pain after bilateral fasciotomy to treat chronic compartment syndrome: a case series study. Journal of Orthopaedic Surgery and Research. [Internet] 2013; 8/1/6: 1-7. Available from: www.josr-online.com/content/8/1/6

[9] Simmonds N, Miller P, Gemmel H. A theoretical framework for the role of fascia in manual therapy. Journal of Bodywork and Movement Therapies. 2012; 16(1): 83-93. PMid: 22196432. http://dx.doi.org/10.1016/j.jbmt.2010.08.001

[10] Didriksen A, Hagen H. Results of chiropractic treatment on 46 patients referred from an ear, nose and throat department. Clinical Chiropractic. 2010: 261-267. http://dx.doi.org/10.1016/j.clch.2010.10.004

[11] Dagens Medicin. Specialuddannede kiropraktorer indtager sygehusene. [Internet] 2011. Available from: http://www.dagensmedicin.dk/nyheder/nordisk-institut-for-kiropraktik- og-klinisk-biomekanik-ansatter-direktor/

[12] Nordstrand Blad. Trenger du en lege-kiropraktor. [Internet]. 2011. Available from: http://www.noblad.no/nyheter/trenger-du-en-lege-kiropraktor-1.6371621

[13] Dagens Medicin. Kiropraktorer kan gøre nytte på hospitalerne. [Internet]. 2007. Available from: http://www.dagensmedicin.dk/opinion/debat/kiropraktorer-kan-gore-nytte-pa-hospitalerne/ 